\title{
On detection of quasiclassical states
}

\author{
Michał Oszmaniec ${ }^{1}$ and Marek Kuś ${ }^{2}$ \\ Center for Theoretical Physics, Polish Academy of Sciences, Al. Lotników 32/46, \\ 02-668 Warszawa, Poland \\ E-mail: ${ }^{1}$ michal.oszmaniec@gmail.com, ${ }^{2}$ marek.kus@cft.edu.pl
}

\begin{abstract}
We give a criterion of classicality for mixed states in terms of expectation values of a quantum observable. Using group representation theory we identify all cases when the criterion can be computed exactly in terms of the spectrum of a single operator.
\end{abstract}

PACS numbers: 03.65.Aa, 02.20.Sv, 03.65.Sq, 03.67.Mn

AMS classification scheme numbers: 81R30, 81P40, 81R05

Submitted to: J. Phys. A: Math. Gen.

\section{Introduction}

Group theoretical coherent states are generalizations of the well known coherent states of a quantum harmonic oscillator [1]. Loosely speaking they appear when an underlying symmetry group $K$ (which is usually a Lie group) is represented in some Hilbert space $V$. The action of $K$ on a vector induces an overcomplete set of vectors in $V$. Standard coherent states of a harmonic oscillator are obtained from the action of the Heisenberg group in $L^{2}(\mathbb{R})$. The set of coherent states possess also many other classical features [1, 2] provided the group $K$ is semisimple, compact and one considers coherent states that contain the vector of the highest weight. We shall call such states quasiclassical or, for shortness, simply 'classical'. They find applications in many branches of modern quantum physics ranging from quantum optics, quantum statistical mechanics, quantum chaos to investigations of classical limit of quantum systems [3].

The 'classicality' of coherent states can manifest itself in several different aspects. Probably the most important facet is that they minimize appropriate uncertainty relations [1, 4] and as such they resemble in the most optimal way classical distributions in the phase space. In the theory of entanglement separable states of multicomponent systems, i.e., states exhibiting only classical correlations can be also treated as coherent states for groups of 'local' transformations. Adapting the notion of 'locality' to a situation at hand, which on the formal level reduces to a proper choice of an underlying 
group and its representation, it is possible to give an unified treatment of entanglement not only for distinguishable particles but also for bosonic and fermionic systems [2, 5].

In the general case of semisimple compact groups it is fairly easy to characterize the set of pure classical via a simple algebraic criterion [2, 6]. It can be cast in the form of the expectation value of an observable (a Hermitean operator) and as such can be, in principle, a basis for experimental check of classicality.

The concept of classical states can be extended onto mixed states by taking convex combinations of pure classical states [7]. For mixed states the problem of finding sufficient and necessary criteria of classicality remains essentially open. In fact for separable states as well as for spin coherent states such criteria are available only in lowdimensional cases [7, 8, 9]. The common origin of these criteria, as well as reformulation of them in terms of expectation values of observables was discussed in [2] and explained in terms of properties of generalized coherent states.

The aim of the paper is to provide methods and framework to quantify the classicality of mixed states from the perspective of the representation theory of semisimple Lie groups and to give a group theoretic characterization of cases when it is possible to give an explicit, closed form criterion for a mixed state to be classical.

The article is organized as follows. In the following section we introduce some basic notions of representation theory of semisimple Lie groups. In the third section we define classical states and present their purely algebraic characterization due to Lichtenstein [6]. Subsequently in Section 4 we recall briefly Jamiołkowski-Choi isomorphism which connects completely positive maps on $V$ and nonnegative operators on $V \otimes V$. We shall make use of this formalism in the rest of the paper. In Section 5 we define classical mixed states and extend an algebraic criterion of classicality on this class of states with the help of the "convex roof" construction. We also discuss known examples in which it is possible to compute appropriate convex roof exactly. In each of those examples exact computation is possible because classicality of pure states is described by an equation involving particular antiunitary operator. In Section 6 we generalize these cases by introducing "antiunitary operators detecting classicality". This part contains our main findings, we characterize cases in which such antiunitaries can occur. Firstly, they appear if and only if a symmetric product $V \vee V$ decomposes onto two irreducible representations from which one is a trivial, one-dimensional one. Secondly, it turns out that there is a relation between the existence of such antiunitaries and the existence of an epimorphism (i.e. an onto homomorphism) of the group $K$ onto one of three groups: special orthogonal group $S O(N)$ (here $N=\operatorname{dim}(V)$ ), exceptional Lie group $G_{2}$ [17] or $\operatorname{Spin}(7)$.

\section{Elements of representation theory}

Let us start with collecting some basic notions and facts from the representation theory of Lie groups and Lie algebras (see eg., [10, 11]).

In the following $K$ is a compact, simply connected and semisimple Lie group, and 
$\mathfrak{k}$ its (real) Lie algebra (compact and semisimple). Due to simple connectedness of $K$ representations of $K$ and $\mathfrak{k}$ are in one to one correspondence. Due to compactness of $K$ every complex representation of $K$ (and thus also of $\mathfrak{k}$ ) is simply reducible.

We denote by $G=K^{\mathbb{C}}$ and $\mathfrak{g}=\mathfrak{k}^{\mathbb{C}}$ the complexifications of the above algebraic structures, thus $\mathfrak{g}$ is a semisimple complex Lie algebra. Two properties of representations mentioned above are also inherited by $G$ and $\mathfrak{g}$.

Let $\mathfrak{t} \subset \mathfrak{k}$ be some (fixed) Cartan subalgebra of $\mathfrak{k}$ i.e., a maximal abelian subalgebra of $\mathfrak{k}$ having self-normalizing property, (for $T \in \mathfrak{t}$ we have that $[T, X] \in \mathfrak{t}$ implies $X \in \mathfrak{t}$ ). Let also $\mathfrak{h}=\mathfrak{t}^{\mathbb{C}}$ be the complexification of $\mathfrak{t}$.

The group $G$ acts in a natural way on its algebra via the adjoint representation Ad $: G \rightarrow G L(\mathfrak{g}), \operatorname{Ad}_{g}(X)=g X g^{-1}, g \in G, X \in \mathfrak{g}$. By differentiation it induces the adjoint representation of $\mathfrak{g}$, ad $: \mathfrak{g} \rightarrow \mathfrak{g l}(\mathfrak{g}), \operatorname{ad}_{Y}(X)=[Y, X], X, Y \in \mathfrak{g}$. Due to semisimplicity of $\mathfrak{g}$ it decomposes as $\mathfrak{g}=\mathfrak{h} \oplus \bigoplus_{\alpha} \mathfrak{g}_{\alpha}$, where the subspace $\mathfrak{g}_{\alpha}$ is spanned by $X$ such that there exist an element $\alpha \in \mathfrak{h}^{*}$ (the space dual to $\mathfrak{h}$ ) for which $\operatorname{ad}_{H}(X)=\alpha(H) X$. Such $\alpha \in \mathfrak{h}^{*}$ are called roots whereas $\mathfrak{g}_{\alpha}$ are called root spaces. It turns out that the root spaces are one dimensional.

It is possible to chose the basis of $\mathfrak{h}^{*}$ (basis elements of this kind are called positive simple roots) in such a way that all roots can be expressed either as a positive integer combination of basis elements (such roots are called positive $-\mathfrak{n}_{+}$) or as a negative integer combination of them (such roots are called negative $-\mathfrak{n}_{-}$). Therefore the root decomposition of $\mathfrak{g}$ can be rewritten as $\mathfrak{g}=\mathfrak{n}_{-} \oplus \mathfrak{h} \oplus \mathfrak{n}_{+}$. One checks that the Lie subalgebras $\mathfrak{n}_{+}$and $\mathfrak{n}_{-}$are nilpotent.

In what follows we denote by $V$ a finite-dimensional complex vector space on which $K$ and $\mathfrak{k}$ are irreducibly represented via complex representations $\Pi$ and $\pi$, respectively. $\Pi$ and $\pi$ extend uniquely to representations of $G$ and $\mathfrak{g}$ on $V$ (which will be denoted by the same symbols).

A convenient way of description of representations of $\mathfrak{g}$ uses the notion of weights vectors, i.e., simultaneous eigenvectors of representatives of all elements form the Cartan subalgebra $\mathfrak{h}$. It means that $v_{\lambda} \in V$ is a weight vector if $\pi(H) v_{\lambda}=\lambda(H) v_{\lambda}$ for $H \in \mathfrak{h}$, where a form $\lambda \in \mathfrak{h}^{*}$ is called the weight of $\pi$. Since we assume that $\mathfrak{g}$ is semisimple and $V$ is a carrier space of an irreducible representation, $V$ decomposes as $V=\oplus_{\lambda} V_{\lambda}$, where summation is over all weights of the considered representation. The subspaces $V_{\lambda}$ are called weight spaces and are spanned by the corresponding weight vectors $v_{\lambda}$. An irreducible representation is uniquely characterized by its highest weight $\lambda_{0}$ determined by the highest weight vector $v_{\lambda_{0}}$, i.e., by the (unique, up to multiplicative constant) weight vector annihilated by all representatives of $n_{+}, \pi\left(\mathfrak{n}_{+}\right) v_{\lambda_{0}}=0$. We will write $V^{\lambda_{0}}$ instead of $V$ when we want to distinguish which irreducible representation of $\mathfrak{g}$ is considered.

Since $\mathfrak{g}$ is semisimple its Killing form $B: \mathfrak{g} \times \mathfrak{g} \rightarrow \mathbb{C}, B(X, Y)=\operatorname{tr}\left(\operatorname{ad}_{X} \circ \operatorname{ad}_{Y}\right)$ is nondegenerate and establishes a correspondence between $\mathfrak{g}$ and $\mathfrak{g}^{*}, \tilde{B}: \mathfrak{g} \rightarrow \mathfrak{g}^{*}$, $\tilde{B}(X)=K(X, \cdot) \in \mathfrak{g}^{*}$, intertwining adjoint and coadjoint representations, $\operatorname{Ad}_{g}^{*}(\tilde{B}(X))=$ $\tilde{B}\left(\operatorname{Ad}_{g}(X)\right)$. 
Let $X_{i}$ form a basis in $\mathfrak{g}$. An element of the universal enveloping algebra $\mathfrak{U}(\mathfrak{g})$ (see [11] for the definition of $\mathfrak{U}(\mathfrak{g}))$ defined as $C_{2}=\sum_{i} \sum_{j} B^{i j} X_{i} X_{j}$, is called the secondorder Casimir operator of the group $K$. Here $B^{i j}=\left(B^{-1}\right)_{i j}$ is the inverse matrix of the Killing form, $B_{i j}=B\left(X_{i}, X_{j}\right)$. It can be checked that $C_{2}$ commutes with all $X_{i}$ and therefore belongs to the center of $\mathfrak{U}(\mathfrak{g})$. As a result $C_{2}$ acts as a multiplication by a scalar on every irreducible representation of $\mathfrak{g}$ (the scalar depends upon the considered representation). A basis of $\mathfrak{g}$ can be chosen in a convenient way (see [11]), $B\left(H_{i}, H_{j}\right)=$ $\delta_{i j}, B\left(X_{\alpha}, X_{-\alpha}\right)=1$, where $H_{i}$ compose a basis in $\mathfrak{h}$ whereas the vectors $X_{\alpha}$ span the corresponding (one-dimensional) root spaces $\mathfrak{g}_{\alpha}$. All other cross-products vanish. In consequence $X_{\alpha}$ and $X_{-\alpha}$ compose bases in, respectively, $\mathfrak{n}_{+}$and $\mathfrak{n}_{-}$. In this basis the second-order Casimir element reads as $C_{2}=\sum_{\alpha>0}\left(X_{-\alpha} X_{\alpha}+X_{\alpha} X_{-\alpha}\right)+\sum_{i} H_{i}^{2}$. It is a known fact that $\left[X_{\alpha}, X_{-\alpha}\right]=B\left(X_{\alpha}, X_{-\alpha}\right) H_{\alpha}$, where $H_{\alpha}$ is an element to which the root $\alpha \in \mathfrak{h}^{*}$ is dual under the action of $\left.\tilde{B}\right|_{\mathfrak{h}}$. As a result, when $\pi: \mathfrak{g} \rightarrow \mathfrak{g l}\left(V^{\lambda_{0}}\right)$ is an irreducible representation of $\mathfrak{g}$ with the highest weight $\lambda_{0}$, we have $\pi\left(C_{2}\right)=\left(\lambda_{0}+2 \delta, \lambda_{0}\right) \mathbb{I}=l\left(\lambda_{0}\right) \mathbb{I}$, where $(\cdot, \cdot): \mathfrak{h}^{*} \times \mathfrak{h}^{*} \rightarrow \mathbb{C}$ is the $\mathrm{Ad}^{*}$-invariant scalar product on $\mathfrak{h}^{*}$ defined by the Killing form and $\delta=\frac{1}{2} \sum_{\alpha>0} \alpha$.

\section{Pure classical states and their algebraic characterization}

As stated in the Introduction by classical (pure) states we understand a special class of generalized coherent states. The latter are defined in terms of an irreducible representation of a group and the 'origin' - a chosen vector in the representation space. More precisely, let $\Pi: K \rightarrow \operatorname{End}(V)$ be an irreducible, unitary representation of a Lie group $K$. We fix a vector $v_{0} \in V$ of the unit length $\left(\left(v_{0} \mid v_{0}\right)=1\right)$ and define the a manifold of coherent states as an orbit through $v_{0}$,

$$
\mathcal{O}_{v_{0}}=\left\{v(k)=\Pi(k) v_{0} \mid k \in K\right\} .
$$

From the quantum mechanical point of view it is more appropriate to consider action of $K$ on the projective space $\mathbb{P} V$ rather than on $V$ itself, as in the physical interpretation of vectors from $V$ their phase does not play a role and we use only vectors normalized to unity. The group $K$ acts naturally on $\mathbb{P} V$ by projection of the representation $\Pi$ : $\tilde{\Pi}(k)[v]=[\Phi(k) v],[v] \in \mathbb{P} V([\cdot]: V \rightarrow \mathbb{P} V$ is a canonical projection of $V$ onto its projective space). Elements of $\left[\mathcal{O}_{v_{0}}\right]=\tilde{\Pi}(K)\left(v_{0}\right)$ are called generalized coherent states (with respect to the representation $\Pi$ ) of the Lie group $K$ with the origin at $v_{0}$ [1]. If $\Pi$ is an irreducible representation with the highest weight $\lambda_{0}$ and corresponding weight vector $v_{\lambda_{0}}$, the elements of $\tilde{\Pi}(K)\left(v_{\lambda_{0}}\right)$ are called 'coherent states closest to the classical' [1]. As explained above we will call them simply (pure) classical states. They can be physically interpreted as orthogonal projections on all pure states generated by action of $K$ on $V^{\lambda_{0}}$. The set of pure classical states will be denoted $C S_{K}\left(V^{\lambda_{0}}\right)$.

There exists a simple, purely algebraic characterization of the set of classical states, i.e., in the group representation language, the orbit through the highest weight vector, given by Liechtenstein [6]. Let $L: V^{\lambda_{0}} \vee V^{\lambda_{0}} \rightarrow V^{\lambda_{0}} \vee V^{\lambda_{0}}$ represents the second order 
Casimir $\left(C_{2}\right)$ of $\mathfrak{g}$ on the symmetrization $V^{\lambda_{0}} \vee V^{\lambda_{0}}$ of $V^{\lambda_{0}} \otimes V^{\lambda_{0}}$, achieved by extending representation $\pi \otimes \mathbb{I}+\mathbb{I} \otimes \pi$ of $\mathfrak{g}$ to representation of $\mathfrak{U}(\mathfrak{g})$ ). The result of Lichteinstein states that

$$
[v] \in C S_{K}\left(V^{\lambda_{0}}\right) \Longleftrightarrow L(v \otimes v)=\left(2 \lambda_{0}, 2 \lambda_{0}+\delta\right) v \otimes v=l\left(2 \lambda_{0}\right) v \otimes v .
$$

Since $K$ is compact all finite dimensional representations of its complexification $G$ (and therefore also of its Lie algebra $\mathfrak{g}$ ) are reducible. In particular, the symmetric tensor product decomposes into a direct sum of irreducible representations. One of these is always the representation $V^{2 \lambda_{0}}$, and in fact this the one with the largest value $C_{2}$, hence we can write

$$
V^{\lambda_{0}} \vee V^{\lambda_{0}}=V^{2 \lambda_{0}} \oplus \underset{\beta<2 \lambda_{0}}{\bigoplus^{\beta}}
$$

where $\beta<2 \lambda_{0}$ means that the summands correspond to values of $C_{2}$ smaller than that for $V^{2 \lambda_{0}}$ )

Therefore,

$$
L=l\left(2 \lambda_{0}\right) \mathbb{P}_{2 \lambda_{0}} \oplus \bigoplus_{\beta<2 \lambda_{0}} l(\beta) \mathbb{P}_{\beta},
$$

where $\mathbb{P}_{(\cdot)}$ denote projections onto appropriate subspaces of $V^{\lambda_{0}} \vee V^{\lambda_{0}}$. One can therefore reformulate Lichteinstein theorem as follows,

$$
[v] \in C S_{K}\left(V^{\lambda_{0}}\right) \Longleftrightarrow \mathbb{P}_{2 \lambda_{0}}(v \otimes v)=v \otimes v \Longleftrightarrow v \otimes v \in V^{2 \lambda_{0}} .
$$

It is therefore very easy to check whether a given state is classical. All one has to do is to verify whether $v \otimes v \in V^{2 \lambda_{0}}$.

\section{CP maps, Jamiołkowski - Choi isomorphism and Kraus decomposition}

In the following we will make use of some techniques which, to our knowledge, were rarely employed in the theory of coherent states. They belong to linear algebra and are of fundamental relevance in quantum information theory, or more precisely, the theory of entanglement. Although original motivations behind the concepts we are going to employ are of no importance for our goals, we found it expedient to present them to some extend to make the techniques more accessible.

A state of a quantum system interacting with an environment is described by its density matrix $\rho$, i.e., a positive semi-definite, and thus necessarily Hermitian, linear operator acting on the Hilbert space of the system. In order to be able to calculate probabilities of events and quantum averages (expectation values) of observables we impose an additional condition of the unit trace, $\operatorname{tr} \rho=1$. Whatever happens to a state during its evolution, the defining properties of positive semi-definiteness and normalization must be retained if the evolved state has to be interpreted as some density matrix. Thus a minimal condition which must be fulfilled by an operator $\Lambda$ representing quantum evolution (such an operator acts in the space of density matrices, or more generally in the space of linear operators on the original Hilbert space) is that 
it transforms positive semi-definite operators into like ones, i.e., it preserves positivity. Such operators (the notion of map is commonly used in this context) are called positive. A moment of reflection suffices to realize that positivity is only a necessary but not sufficient condition requested from a map representing quantum evolution. Indeed, the system in question can be always treated as a part of a larger one consisting of it and some environment. The density of state of the compound system must also evolve keeping the positive semi-definiteness intact, even if 'nothing happens' to the environment itself. It means that tensoring $\Lambda$ with the identity (representing lack of actual evolution of the environment) must also produce a positive map acting on density states of the system plus environment. A map $\Lambda$ fulfilling this condition is called completely positive.

The formal definition reads,

Definition 4.1. Let $V$ be a complex finite-dimensional Hilbert space and denote by B(V) the space of linear operators on $V$. A completely positive map on $V$ (in short $C P$ map on $V)$ is a linear mapping $\Lambda: B(V) \rightarrow B(V)$ between sets of linear operators on $V$ that,

- Preserves hermiticity, $A=A^{\dagger} \Rightarrow \Lambda(A)=\Lambda(A)^{\dagger}$.

- Preserves positivity of operators, $\Lambda \geq 0 \Rightarrow \Lambda(A) \geq 0$. i.e., $\Lambda$ is a positive map.

- The map $\mathbb{I}_{p} \otimes \Lambda$, where $\mathbb{I}_{p}$ is the $p \times p$ identity matrix, is positive for arbitrary $p$.

The set of CP maps on a Hilbert space $V$ will be denoted $C P(V)$.

To make the introduced concept useful in applications we need two things, a practical criterion allowing for an easy check whether a map is completely positive and, possibly, a description of the structure of completely positive maps. The former is based on an interesting connection between positive bilinear operators on some Hilbert space $V$, i.e., positive semi-definite linear operators on $V \otimes V$, the set of which we will denote by $P(V \otimes V)$ and (completely) positive maps on the space of linear operators on $V$. It is given by the so called Choi-Jamiołkowski isomorphism which we now briefly describe.

Theorem 4.1. (Jamiotkowski-Choi [12]) There is one to one correspondence between completely positive maps on $N$-dimensional complex Hilbert space $V$ and positive operators on $V \otimes V$. The isomorphism is given by the Jamiotkowski mapping $J$ : $C P(V) \rightarrow P(V \otimes V)$

$$
J(\Lambda)=\left(\mathbb{I}_{N} \otimes \Lambda\right)(\mid \Phi)(\Phi \mid),
$$

where $\Lambda \in C P(V), \Phi=\sum_{i=1}^{i=N} e_{i} \otimes e_{i}$ is the maximally entangled state in $\mathcal{H}$, and $\left\{e_{i}\right\}_{i=1}^{i=N}$ is some fixed orthogonal basis in $V$.

Checking the complete positivity can be thus reduced to determining whether the corresponding operator $J(\Lambda)$ acting on $V \otimes V$ is positive semi-definite which can be easily achieved by the spectral decomposition of $J(\Lambda)$.

In the applications that follow we will use the reasoning going in the reverse direction. Knowing that a bilinear operator on $V \otimes V$ is positive semi-definite we infer that the corresponding map $\Lambda$ is completely positive. To this end we need the following 
Theorem 4.2. The inverse of the Jamiotkowki map $J^{-1}: P(V \otimes V) \rightarrow C P(V)$ is given by

$$
\left(J^{-1}(A)\right)(\rho)=\operatorname{tr}_{1}\left[\left(\rho^{T} \otimes \mathbb{I}_{N}\right) A\right],
$$

where $A \in P(V \otimes V), \rho \in B(V), \operatorname{tr}_{1}: B(V \otimes V) \rightarrow B(V)$ is the partial trace over the first Hilbert space, and $\rho^{T}$ is the transpose of the operator $\rho$ in the basis $\left\{e_{i}\right\}_{i=1}^{i=M}$.

The second ingredient important in our argumentation is the above mentioned structural characterization of completely positive maps. It is provided by the fact that each CP map allows a so called Kraus decomposition [12].

Theorem 4.3. For each $\Lambda \in C P(V)$ there exists a set of operators $T_{\alpha}: V \rightarrow V(\alpha \in \mathcal{A}$, where $\mathcal{A}$ is some set of indices) such that for all $\rho \in B(V)$,

$$
\Lambda(\rho)=\sum_{\alpha \in \mathcal{A}} T_{\alpha} \rho T_{\alpha}^{\dagger}
$$

The form of $\Lambda$ given by Equation (8) is called its Kraus decomposition with Kraus operators $T_{\alpha}$.

The Kraus decomposition is not unique yet there is a distinguished one associated with the spectral decomposition of $J(\Lambda)=A$. If $\left\{f_{\alpha}\right\}_{\alpha \in \mathcal{A}}$ is the orthonormal basis of eigenvectors of $A$ that correspond to eigenvalues $\left\{\lambda_{\alpha}\right\}_{\alpha \in \mathcal{A}}$, we define,

$$
T_{\alpha}=\lambda_{\alpha}^{\frac{1}{2}}\left(\Phi \mid \otimes \mathbb{I}_{N}\right)\left(\mathbb{I}_{N} \otimes \mid f_{\alpha}\right) .
$$

The notation used in in the above formula, although commonly used, probably needs some elucidation. Observe that both $\left(\Phi \mid\right.$ and $\left.\mid f_{\alpha}\right)$ are linear combinations of simple tensors (the former from its definition, the latter as an eigenvector of $A$ acting in the tensor product $V \otimes V)$. For simple tensors the corresponding formula reads $\left.\left(e_{i}\left|\otimes\left(e_{i} \mid \otimes \mathbb{I}_{N}\right)\left(\mathbb{I}_{N} \otimes \mid a\right) \otimes\right| b\right)=\left(e_{i} \mid a\right) \mid b\right)\left(e_{i} \mid\right.$, which is indeed a linear operator on $V$.

It turns out 12 that $T_{\alpha}$ form indeed Kraus decomposition of $\Lambda$. The importance of this particular Kraus decomposition is twofold. Firstly, operators $T_{\alpha}$ are orthogonal to each other with respect to standard Hilbert-Schmidt product on $B(V)$. Secondly, the cardinality of $\mathcal{A}$ is minimal. It is possible to express matrix coefficients of any $A \in P(V \otimes V)$ in terms of operators from Kraus decomposition of the CP map corresponding to it. It can be proved [2] that,

$$
\left(v_{1} \otimes v_{2} \mid A\left(v_{3} \otimes v_{4}\right)\right)=\sum_{\alpha \in \mathcal{A}}\left(v_{1} \mid T_{\alpha} \mathcal{K} v_{2}\right)\left(\mathcal{K} v_{3} \mid T_{\alpha}^{\dagger} v_{4}\right),
$$

where $v_{1}, v_{2}, v_{3}, v_{4} \in V$ and $\mathcal{K}$ is the complex conjugation of a vector expressed in the base used to define Jamiołkowski isomorphism, $\mathcal{K}\left(\sum_{i=1}^{i=N} v^{i} e_{i}\right)=\sum_{i=1}^{i=N} \overline{v^{i}} e_{i}$.

In physical applications an important class of $\mathrm{CP}$ maps is the class of so called quantum channels, i.e., CP maps that preserve traces. A map $\Lambda$ is a quantum channel if $\operatorname{tr}[\Lambda(\rho)]=\operatorname{tr}[\rho]$. On the level of Kraus decomposition of $\Lambda$ this condition reduces to the requirement that, $\sum_{\alpha \in \mathcal{A}} T_{\alpha} T_{\alpha}^{\dagger}=\mathbb{I}_{N}$. How is this condition realized on the level of the operator $A=J(\Lambda) \in P(V \otimes V)$ ? It is easy to check (see Eq. (77) and Eq. (8)) that it 
is necessary and sufficient to have $\operatorname{tr}_{1}[A]=\mathbb{I}_{N}$. In this article we focus on the situation when we have some nonnegative $A$ with only one nonzero eigenvalue. As discussed above this situation allows to chose only one Kraus operator in the decomposition of the corresponding $\Lambda$. If we assume that $\Lambda$ is a quantum channel we get that the corresponding Kraus operator $T_{\alpha_{0}}$ is unitary,

$$
T_{\alpha_{0}} T_{\alpha_{0}}^{\dagger}=\mathbb{I}_{N}
$$

Note that if $T_{\alpha_{0}} T_{\alpha_{0}}^{\dagger} \propto \mathbb{I}_{N}$ one can rescale the initial $A\left(A \rightarrow A^{\prime}=c A\right)$ so that resulting $T_{\alpha_{0}}^{\prime}$ is unitary. By the virtue of Eq.(10) in the case of unitary $T_{\alpha_{0}}$ expectation value of $A$ can be expressed in terms of antiunitary operator $\theta=T_{\alpha_{0}} \mathcal{K}$,

$$
(v \otimes v \mid A(v \otimes v))=|(v \mid \theta v)|^{2} .
$$

This observation will turn out to be crucial during the discussion of classical mixed states.

\section{Mixed classical states and their characterization}

The definition of classical states is extended to the case when state of a system is described by a density matrix [2, 7].

Definition 5.1. The set of mixed classical states on $V^{\lambda_{0}}$, denoted in the following by $M C S_{K}\left(V^{\lambda_{0}}\right)$ ) consists of mixed states that can be expressed as a convex combination of projections on pure classical states,

$$
\left.\rho \in M C S_{K}\left(V^{\lambda_{0}}\right) \Longleftrightarrow \rho=\sum_{i} p_{i} \mid v_{i}\right)\left(v_{i} \mid, \sum_{i} p_{i}=1, p_{i} \geq 0,\left[v_{i}\right] \in C S_{K}\left(V^{\lambda_{0}}\right) .\right.
$$

To treat pure and mixed states on the same footing we may identify a pure state $[v]$ with the projection on $v$, i.e., $[v] \sim \mid v)\left(v \mid\right.$. The set of mixed classical states, $M C S_{K}\left(V^{\lambda_{0}}\right)$, is in this language the convex hull of the pure classical states.

In order to detect effectively classical states it would be desirable to find an extension of the Liechtenstein criterion to to mixed states. Unfortunately no such extension is known. One way of attacking the problem consists of employing the so called convex roof construction. To do this we need some results from convex geometry. Let $W$ be a real, finite dimensional vector space. If $S$ is an arbitrary subset of $W$, by $\operatorname{conv}(S)$ we denote convex hull of $S$, i.e., the set composed of all convex combinations of points from $S$, thus conv $(S)$ is the smallest convex set containing $S$. If $S$ is compact then $\operatorname{conv}(S)$ is also compact. Let $C$ be a convex compact subset of $W$ and let $E$ be the set of its extremal points, i.e., points that do not lie in the interior of any line segment contained in $C$. By the Krein-Milman theorem $C=\operatorname{conv}(E)$. If $f: E \rightarrow \mathbb{R}$ is a continuous function we define its convex roof $f^{\cup}$ extension [13, 14],

$$
f^{\cup}(x)=\inf _{\sum_{k} p_{k} x_{k}=x} \sum_{k} p_{k} f\left(x_{k}\right), x \in C, x_{k} \in E,
$$

where the infimum is taken over all possible convex decompositions of $x$ onto vectors from the set of extremal points $E$. In the following we shall make use of the properties of $f^{\cup}$ outlined by the theorem (see [13, 14]), 
Theorem 5.1. Function $f^{\cup}$ is convex in $C$. Moreover, $f^{\cup}$ is the smallest convex extension of $f$ (i.e. smallest convex function that coincides with $f$ on $E$ ).

Let $E_{0} \subset E$ be some compact subset of the set of extremal points and let $\operatorname{conv}\left(E_{0}\right) \subset C$ be its convex hull. If $\left.f\right|_{E_{0}}=c$ and $\left.f\right|_{E \backslash E_{0}}>c$, then $f^{\cup}(x)=c$ if and only if $x \in \operatorname{conv}\left(E_{0}\right)$. Therefore $f^{\cup}$ can serve as an identifier of the set $\operatorname{conv}\left(E_{0}\right)$.

Let us come back to our main considerations. In our framework mixed states $M S$ correspond to $C$ whereas pure states correspond to $E$. Pure classical states $C S_{K}\left(V^{\lambda_{0}}\right)$ play the role of $E_{0}$ and therefore mixed classical states $M C S_{K}\left(V^{\lambda_{0}}\right)$ can be identified with $\operatorname{conv}\left(E_{0}\right)$. By constructing appropriate function on the set of pure states it is possible to construct a characterization of mixed classical states. Let us define,

$$
f_{1}(\mid \phi)(\phi \mid)=\sqrt{\left(\phi \otimes \phi\left|\left(\mathbb{I} \otimes \mathbb{I}-\mathbb{P}_{2 \lambda_{0}}\right)\right| \phi \otimes \phi\right)},
$$

where $\mathbb{P}_{2 \lambda_{0}}$ is the projection onto the representation with the highest weight $2 \lambda_{0}$ embedded in $V^{\lambda_{0}} \vee V^{\lambda_{0}}$ (see Eq. (3) ). It is clear that $f_{1}$ is well defined and continuous and reaches the minimum (equal to 0) on the set of pure classical states $C S_{K}\left(V^{\lambda_{0}}\right.$ ) (see Eq.(5) ). Therefore $f_{1}^{\cup}$ will distinguish between classical and nonclassical mixed states. Due to the fact that $f_{1}$ is $\frac{1}{2}$ - homogenous we can write,

$$
f_{1}^{\cup}(\rho)=\inf _{\left.\sum_{k} \mid v_{k}\right)\left(v_{k} \mid=\rho\right.} \sum_{k} \sqrt{\left(v_{k} \otimes v_{k}\left|\left(\mathbb{I} \otimes \mathbb{I}-\mathbb{P}_{2 \lambda_{0}}\right)\right| v_{k} \otimes v_{k}\right)} .
$$

Here the infimum is taken over all decompositions of $\rho$ into a sum of operators of rank one (not necessary normalized). In general the infimum in the formula for $f_{1}^{\cup}$ cannot be computed explicitly for arbitrary $\rho$, one has then rely on various, relatively easily computable estimates, which, however, give only sufficient criteria of non-classicality [15] leaving a margin of uncertainty in discriminating mixed classical states. There are however cases when the effective computation of the infimum is possible [13]. They correspond to situations when the operator expectation value of $\mathbb{I} \otimes \mathbb{I}-\mathbb{P}_{2 \lambda_{0}}$ can be expressed in terms of some antiunitary operator $\tilde{\theta}$ (note that at this point $\tilde{\theta}$ can be different from $\theta$ in Eq.(12)) in the following way,

$$
\left(v \otimes v \mid\left(\mathbb{I} \otimes \mathbb{I}-\mathbb{P}_{2 \lambda_{0}}\right)(v \otimes v)\right)=|(v \mid \tilde{\theta} v)|^{2} .
$$

In such situations we have,

$$
f_{1}^{\cup}(\rho)=\inf _{\left.\sum_{k} \mid v_{k}\right)\left(v_{k} \mid=\rho\right.} \sum_{k}\left|\left(v_{k} \mid \tilde{\theta} v_{k}\right)\right|,
$$

and we can perform the minimization [13],

$$
f_{1}^{\cup}(\rho)=\max \left\{0, \mu_{1}-\sum_{j=2}^{r} \mu_{j}\right\},
$$

where $\left\{\mu_{j}\right\}_{j=1}^{j=r}$ are increasingly ordered eigenvalues of the operator $|\sqrt{\rho} \tilde{\theta} \sqrt{\rho}|$. According to our knowledge situations expressed by Eq.(17) are the only ones in which in is possible to compute $f_{1}^{\cup}$ explicitly. The list of known examples of this kind described in literature [2, 9] is short and contains only three examples, 
(i) Three-dimensional (labeled by spin $S=1$ ) representation of $K=S U(2)$. It is a know fact that $V^{1} \vee V^{1}=V^{2} \oplus V^{0}$, where $V^{0}$ is the one-dimensional trivial representation (labeled by spin $S=0$ ) and $V^{2}$ is the five-dimensional representation (labeled by spin $S=2$ ). This representation is used used in the description of two bosons of $\operatorname{spin} S=1$.

(ii) Four-dimensional representation of $S U(2) \times S U(2)$ defined by its natural action on $\mathcal{H}=\mathbb{C}^{2} \otimes \mathbb{C}^{2}$. This representation is used to describe entanglement of two qubits [2, 8].

(iii) Six-dimensional representation of $S U(4)$ labeled by highest weight $(1,1,0)$ (for more details concerning notation see [16]). Representation $V^{(1,1,0)}$ is isomorphic to the six-dimensional representation of $S U(4)$ acting on $\mathbb{C}^{4} \vee \mathbb{C}^{4}$. This representation is natural for the description of the entanglement of two fermions with spin $S=\frac{3}{2}$.

It is important to note that in each of those cases there exists an epimorphism of the appropriate group $K$ onto the group $S O(N)$, where $N$ is the dimension of the irreducible representation of $K$. This observation was first made in [2] and it was the actual inspiration for this article. In the next section we prove that these examples are not accidental and are the manifestation of a rather general principle relating epimorphisms of $K$ and some $S O(N)$, antiunitary operators, and the decomposition of the symmetric power of the representation considered onto irreducible components.

\section{Main results}

In this part we characterize in terms of the representation theory of compact semisimple Lie groups all situations in which Eq.(17) takes place and computation of the "nonclassicality witness" $f_{1}^{\cup}$ is possible (see Eq.(19)). Let us first introduce the concept of antiunitary operators that "detect classicality". It will prove to be useful in our considerations.

Definition 6.1. We shall say that an antiunitary operator $\theta: V^{\lambda_{0}} \rightarrow V^{\lambda_{0}}$ detects classicality if it satisfies the following,

- $\theta$ is $K$-invariant, that is, $\Pi(k)^{\dagger} \theta \Pi(k)=\theta$ for each $k \in K$.

- Expectation value of $\theta$ vanishes exactly on classical states, $(v \mid \theta v)=0 \Longleftrightarrow[v] \epsilon$ $C S_{K}\left(V^{\lambda_{0}}\right)$.

We present our results in two theorems. First of them relate the existence of antiunitary operators detecting classicality to the decomposition of $V^{\lambda_{0}} \vee V^{\lambda_{0}}$. Second theorem connects this kind of antiunitary operators with the existence of epimorphisms of the group $K$ onto some orthogonal group.

Theorem 6.1. Let $K$ be the semisimple, compact and connected Lie group. Let $\Pi$ be some irreducible unitary representation of the group $K$ in the Hilbert space $V^{\lambda_{0}}$ with the highest weight $\lambda_{0}$. The following two statements are equivalent, 
1. There exists an antiunitary operator $\theta: V^{\lambda_{0}} \rightarrow V^{\lambda_{0}}$ detecting classicality.

2. $V^{\lambda_{0}} \vee V^{\lambda_{0}}=V^{2 \lambda_{0}} \oplus V^{0}$, where $V^{0}$ is a trivial representation of the group $K$.

Proof. $(1 \rightarrow 2)$ Let $\theta=T \tilde{K}$ where $T$ is an unitary operator and $\tilde{\mathcal{K}}$ is the operator of the complex conjugation is some fixed basis of $V^{\lambda_{0}}$, say $\left\{e_{i}\right\}_{i=1}^{i=N}$. Define an operator $A \in P\left(V^{\lambda_{0}} \otimes V^{\lambda_{0}}\right)$ as an image of the Jamiołkowski map (defined with respect to the basis $\left\{e_{i}\right\}_{i=1}^{i=N}$ ) of the CP map $\Lambda(\rho)=T \rho T^{\dagger}$ (see Eq.(6)) ). It is easy to check that matrix elements of $A$ are given by the following formula (see Eq.(10) and [5])

$$
\left(v_{1} \otimes v_{2} \mid A\left(v_{3} \otimes v_{4}\right)\right)=\left(v_{1} \mid T \tilde{\mathcal{K}} v_{2}\right)\left(T \tilde{\mathcal{K}} v_{3} \mid v_{4}\right)=\left(v_{1} \mid \theta v_{2}\right)\left(\theta v_{3} \mid v_{4}\right) .
$$

We now claim that the operator $A$ is proportional to $\mathbb{I} \otimes \mathbb{I}-\mathbb{P}_{2 \lambda_{0}}$ (when both operators are understood as acting on $\left.V^{\lambda_{0}} \vee V^{\lambda_{0}}\right)$. Indeed, $A$ is symmetric and nonnegative. It is also $K$-invariant due to the $K$-invariance of $\theta$,

$$
\begin{aligned}
& \left(\Pi(k) v_{1} \otimes \Pi(k) v_{2} \mid A\left(\Pi(k) v_{3} \otimes \Pi(k) v_{4}\right)\right)=\left(\Pi(k) v_{1} \mid \theta \Pi(k) v_{2}\right)\left(\theta \Pi(k) v_{3} \mid \Pi(k) v_{4}\right) \\
& =\left(v_{1} \mid \theta v_{2}\right)\left(\theta v_{3} \mid v_{4}\right)=\left(v_{1} \otimes v_{2} \mid A\left(v_{3} \otimes v_{4}\right)\right) .
\end{aligned}
$$

Thus,

$$
A=a_{2 \lambda_{0}} \mathbb{P}_{2 \lambda_{0}} \oplus \bigoplus_{\beta<2 \lambda_{0}} a_{\beta} \mathbb{P}_{\beta},
$$

where the above sum corresponds to the decomposition of $V^{\lambda_{0}} \vee V^{\lambda_{0}}$ onto irreducible components (see Eq.(3)) and $a$ 's are some nonnegative scalars. By definition $A$ has only one eigenvector (see our remarks during the discussion of the CP maps and the Kraus decomposition). Projection on this eigenvector cannot belong to $V^{2 \lambda_{0}}$ because the expectation value of $\theta$ vanish on coherent states. On the ofter hand, by the theorem of Lichtenstein and the properties of $\theta$,

$$
|(v \mid \theta v)|>0 \Longleftrightarrow \bigoplus_{\beta<2 \lambda_{0}} \mathbb{P}_{\beta}(v \otimes v) \neq 0
$$

From this it follows that there is only one $\beta$ in the above sum and the corresponding $\mathbb{P}_{\beta}$ is one dimensional because $A$ has rank one. Therefore we get a one dimensional irreducible representation of group $K$. But this irreducible representation must be a trivial representation due to the fact that $K$ is semisimple.

$(2 \rightarrow 1)$ If $V^{\lambda_{0}} \vee V^{\lambda_{0}}=V^{2 \lambda_{0}} \oplus V^{0}$ operator $\mathbb{I} \otimes \mathbb{I}-\mathbb{P}_{2 \lambda_{0}}=\mathbb{P}_{0}$ (acting on $V^{\lambda_{0}} \vee V^{\lambda_{0}}$ ) has rank one and is nonnegative. If we apply to it the inverse of the Jamiołkowski isomorphism (with respect to some fixed basis $\left\{e_{i}\right\}_{i=1}^{i=N}$ ) we get $\Lambda\left(\mathbb{P}_{0}\right)$ together with the corresponding Kraus operator T (see Eq.(77) and Eq.(8) ). By the Eq.(10) we have,

$$
\left(v_{1} \otimes v_{2} \mid \mathbb{P}_{0}\left(v_{3} \otimes v_{4}\right)\right)=\left(v_{1} \mid T \mathcal{K} v_{2}\right)\left(T \mathcal{K} v_{3} \mid v_{4}\right),
$$

where $\mathcal{K}$ is the complex conjugation in the basis $\left\{e_{i}\right\}_{i=1}^{i=N}$. We claim that the antilinear operator $\theta=T \mathcal{K}$ is proportional to the antiunitary operator detecting classicality. By the equation (24) $\theta$ is $K$-invariant. It follows from the $K$-invariance of $\mathbb{P}_{0}$ and the calculation is essentially the reverse of the calculation in Eq.(21). Because of the 
decomposition $V^{\lambda_{0}} \vee V^{\lambda_{0}}=V^{2 \lambda_{0}} \oplus V^{0}$ and Eq.(24) we have $(v \mid \theta v)=0 \Longleftrightarrow[v] \in$ $C S_{K}\left(V^{\lambda_{0}}\right)$. The only thing that needs be proved is that $T$ can be rescaled to the unitary operator. This follows from the discussion of the relation between nonnegative operators on the product of Hilbert spaces and quantum channels (see Eq.(11)). The necessary and sufficient condition for $T$ to be proportional to the unitary operator is $\operatorname{tr}_{1}\left[\mathbb{P}_{0}\right] \propto \mathbb{I}_{N} . \mathbb{P}_{0}$ is the orthogonal projection onto one dimensional trivial representation $V^{0}$ in the decomposition of $V^{\lambda_{0}} \vee V^{\lambda_{0}}$. It can be thus written in the form of the integral with respect to the Harr measure $\mu$ over the whole $K$ [11],

$$
\mathbb{P}_{0}=\int_{K} \Pi(k) \otimes \Pi(k) d \mu(k) .
$$

As a result we have,

$$
\operatorname{tr}_{1}\left[\mathbb{P}_{0}\right]=\int_{K} \operatorname{tr}[\Pi(k)] \Pi(k) d \mu(k)=\int_{K} \chi_{\lambda_{0}}(k) \Pi(k) d \mu(k),
$$

where $\chi_{\lambda_{0}}(k)$ is the character of the representation $\Pi$. By the general representation theory of compact Lie groups we have (see [1]]),

$$
\int_{K} \chi_{\lambda_{0}}(k) \Pi(k) d \mu(k)=\frac{\mathbb{I}_{N}}{N} .
$$

Therefore the proof in now completed.

Note that in the assumptions of the above theorem there is no reference to the dimension of the considered representation $V^{\lambda_{0}}$. It is nevertheless clear that when $\operatorname{dim}\left(V^{\lambda_{0}}\right)=1$ and $\operatorname{dim}\left(V^{\lambda_{0}}\right)=2$ both statements that are meant to be equivalent are at the same time false.

The above proved theorem states that cases when operator $\mathbb{I} \otimes \mathbb{I}-\mathbb{P}_{2 \lambda_{0}}$ has rank one correspond exactly to the appearance of antiunitaries that detect classicality. As advertised, it turns out that such cases are related to the existence of an epimorphism between the group $K$ and one of three groups: $S O(N)$ ( for $N=\operatorname{dim}\left(V^{\lambda_{0}}\right)$ ), $G_{2}$ or $\operatorname{Spin}(7)$. We formulate this fact in the following theorem.

Theorem 6.2. Let $K$ be a semisimple, compact and connected Lie group. The following two statements are equivalent,

1. There exists an irreducible unitary representation $\Pi$ of the group $K$ in the Hilbert space $V^{\lambda_{0}}$ with the highest weight $\lambda_{0}\left(N=\operatorname{dim}\left(V^{\lambda_{0}}\right)>2\right)$. On $V^{\lambda_{0}}$ there exists an antiunitary operator $\theta: V^{\lambda_{0}} \rightarrow V^{\lambda_{0}}$ detecting classicality.

2. There exists an epimorphism $h: K \rightarrow S O(N)$, or $h: K \rightarrow G_{2}$ (the exceptional Lie group $G_{2}$ [17]) with $N=7$, or $h: K \rightarrow \operatorname{Spin}(7)$ with $N=8$.

Proof. $(1 \rightarrow 2)$ Because $\theta$ is antiunitary it is possible to chose the orthonormal basis $\left\{e_{i}\right\}_{i=1}^{i=N}$ of $V^{\lambda_{0}}$ is such a way that each vector from the basis is an eigenvector of $\theta$ with an eigenvalue 1: $\theta e_{i}=e_{i}, i=1, \ldots, N$. In this basis $\theta$ act as a complex conjugation,

$$
v=\sum_{i=1}^{i=N} v_{i} e_{i} \Longrightarrow \theta v=\sum_{i=1}^{i=N} \bar{v}_{i} e_{i} .
$$


From the $K$-invariance of $\theta$ it follows that in this basis unitary operators defining $\Pi(k)$ are real and therefore also orthogonal. Because $\Pi$ is continuous the image of a connected group $K$ must be connected and therefore $\Pi$ defines a homomorphism $h: K \rightarrow S O(N)$. Note that each representative $v \in V^{\lambda_{0}}$ of a state $[v] \in \mathbb{P} V^{\lambda_{0}}$ can be decomposed into its real and imaginary part, $v=u+i \cdot w$ in the basis $\left\{e_{i}\right\}_{i=1}^{i=N}$ described above, i.e., with the vectors $u$ and $w$ being real linear combinations of basis vectors. For a classical state in such a form we have $0=(v \mid \theta v)=(u \mid u)-(w \mid w)+2 i(u \mid w)$, hence all classical states are represented by vectors $v=u+i \cdot w$, where $(u \mid u)=(w \mid w)=1$ and $(u \mid w)=0$. In particular, $v_{\lambda_{0}}=u_{0}+i \cdot w_{0}$ for some orthogonal and appropriately normalized $u_{0}$ and $w_{0}$. For $\tilde{x} \in C S_{K}\left(v_{\lambda_{0}}\right)$ let $\tilde{v}=\tilde{u}+i \cdot \tilde{w}$ be its representative normalized as above. We claim that $\tilde{u}=\Pi(k) u_{0}$ and $\tilde{w}=\Pi(k) w_{0}$ for some $k \in K$. Indeed, we must have $\tilde{v}=\epsilon \Pi\left(k_{1}\right) v_{\lambda_{0}}$ for some unimodular $\epsilon$ and $k_{1} \in K$. Because representation $\Pi$ is non-trivial we can chose $k_{2} \in K$ such that $\epsilon v_{\lambda_{0}}=\Pi\left(k_{2}\right) v_{\lambda_{0}}$. Therefore, if we take $k=k_{1} k_{2}$, we have the desired result (matrices corresponding to $\Pi(k)$ are real in the considered basis),

$$
\Pi(k) u_{0}+i \cdot \Pi(k) w_{0}=\Pi(k) v_{\lambda_{0}}=\epsilon \Pi\left(k_{1}\right) v_{\lambda_{0}}=\tilde{v}=\tilde{u}+i \cdot \tilde{w} .
$$

Thus, it is possible to generate all pairs of orthonormal vectors by the action of $h(K)$ on vectors $u_{o}$ and $w_{0}$, i.e. $\Pi(K)$ acts transitively on pairs of orthonormal vectors. This fact suffices to prove that $h(K)$ equals $S O(N), G_{2}$ or $\operatorname{Spin}(7)$. In order to see this we refer to the classical result of Montgomery and Samelson [18] that classifies all compact and connected Lie groups acting transitively and effectively on $M$ dimensional spheres. In our case $M=N-1$ and we are interested only in compact and connected matrix groups $(h(K)$ is obviously compact, connected and acts transitively and effectively on the sphere). The list of such groups is short and consists of seven cases: $S O(N)$ itself, its three proper subgroups: $S U\left(\frac{N}{2}\right), S p\left(\frac{N}{4}\right)$, and $S p(1) \times S p\left(\frac{N}{4}\right)$ (where $S p(\cdot)$ denotes a compact symplectic group), $G_{2} \subset S O(7), \operatorname{Spin}(7) \subset S O(8)$, and $\operatorname{Spin}(9) \subset S O(16)$. We first consider last three "exceptional" cases. Groups $G_{2}$, $\operatorname{Spin}(7)$ and $\operatorname{Spin}(9)$ act transitively on respectively 6, 7 and 15 dimensional spheres. Those actions come from the following (faithful) representations: defining representation of $G_{2}, 8$ dimensional spinor representation of $\operatorname{Spin}(7)$ and 16 spinor representation of $\operatorname{Spin}(9)$. Actions of $G_{2}$ and $\operatorname{Spin}(7)$ are transitive on orthonormal pairs of vectors (see [17], p. 32). Therefore those groups are permissible. On the other hand, it is known [20] that the 16 dimensional representation of $\operatorname{Spin}(9)$ does not have the desired property. Let us now consider the special unitary and symplectic subgroups of $S O(N)$. Those groups can appear only when 2 (in the case of $S U\left(\frac{N}{2}\right)$ ) or 4 (in the case of $S p\left(\frac{N}{4}\right)$ and $\left.S p(1) \times S p\left(\frac{N}{4}\right)\right)$ are divisors of $N$. Therefore when $N$ is odd the proof is finished. Now assume that 2 or 4 divide $N$. Since $h(K)$ acts transitively on orthonormal pairs of vectors, a subgroup $\operatorname{stab}\left(u_{0}\right)$ of $h(K)$ that stabilizes the vector $u_{0}$ must act transitively on $S^{N-2} \cong S^{N-1} \cap u_{0}^{\perp}$, where $u_{0}^{\perp}$ is the orthogonal complement of $u_{0}$. We can now apply the theorem of Montgomery and Samelson for the dimension $N-1$. Since $N-1$ is now odd we infer that $\operatorname{stab}\left(u_{0}\right)=S O(N-1)$. As a consequence we have $\operatorname{dim}(h(K)) \geq \frac{(N-1)(N-2)}{2}=\operatorname{dim}(S O(N-1))$. Since the dimensions of $S U\left(\frac{N}{2}\right), S p\left(\frac{N}{4}\right)$ 
and $S p(1) \times S p\left(\frac{N}{4}\right)$ are, respectively, $\frac{N^{2}}{4}-1, \frac{N}{4}\left(\frac{N}{2}+1\right)$ and $\frac{N}{4}\left(\frac{N}{2}+1\right)+3$, we can exclude those groups. At the and we conclude that only possibilities are that $h(K)=S O(N)$, $h(K)=G_{2}($ when $N=7)$ or $h(K)=\operatorname{Spin}(7)$ (when $\left.N=8\right)$.

$(2 \rightarrow 1)$ We treat groups $S O(N), G_{2}$ and $\operatorname{Spin}(7)$ together. We consider defining representations of $S O(N)$ and $G_{2}$ and the 8 dimensional spinor represenation of $\operatorname{Spin}(7)$. We shall show that symmetric powers of those irreducible faithful representations (clearly those are also irreducible representations of the group $K$ ) decompose onto two ingredients: $V^{\lambda_{0}} \vee V^{\lambda_{0}}=V^{2 \lambda_{0}} \oplus V^{0}$. Then, combining this with the theorem 6.1 we conclude the existence of the antiunitary operator $\theta$ that detects classicality for each of considered representations. To prove the above decomposition we notice that each representation respects the Euclidean structure in the relevant $V^{\lambda_{0}}$ (when viewed as subgroups of $S O(N), S O(7)$ and $S O(8)$ accordingly) so $V^{\lambda_{0}} \simeq\left(V^{\lambda_{0}}\right)^{*}$. As a result we have $V^{\lambda_{0}} \vee V^{\lambda_{0}} \simeq V^{\lambda_{0}} \vee\left(V^{\lambda_{0}}\right)^{*} \simeq S \operatorname{End}(V)\left(S \operatorname{End}\left(V^{\lambda_{0}}\right)\right.$ denotes symmetric endomorphisms of $\left.V^{\lambda_{0}}\right)$. There is one distinguished element of $S \operatorname{End}\left(V^{\lambda_{0}}\right)$ that is preserved by the group action: $\mathbb{I}$ - the identity in $V^{\lambda_{0}}$. This element corresponds to the one dimensional invariant subspace subspace $V^{0} . V^{2 \lambda_{0}}$ is realized by the the traceless operators from $S E n d(V)$. It is easy to check that this subspace is an irreducible representation - it follows from the transitivity of the action of the each group on pairs of orthonormal vectors (when treated as a subgroup of $S O(N), S O(7)$ and $S O(8)$ respectively). We have thus proved the decomposition $V^{\lambda_{0}} \vee V^{\lambda_{0}}=V^{2 \lambda_{0}} \oplus V^{0}$ which finishes the proof.

\section{Concluding remarks}

We presented group theoretical conditions for the cases when antiunitary operator detecting classicality exists and it is possible to compute $f_{1}^{\cup}$ exactly. Theorem 6.1 links such cases with situations in which symmetric power of the considered representation decomposes onto two irreducible components one of which is a trivial, one dimensional representation.

In the course of the proof of the Theorem 6.2 we referred to the classical work by Montgomery and Samelson [18. Although it may seem to be a trick from a rather 'high floor' we would like to stress that the problem is not as easy as it may seem at the first sight. It turns out that when $N$ is even there are proper subgroups of $S O(N)$ that act transitively on $S^{N-1}$ (it turns out that this fact is directly related to the classification of the holonomy groups of irreducible non locally symmetric Riemannian spaces [19]). Nevertheless our assumption about the existence of an 'antiunitary operator detecting classicality' is strong enough to guarantee that the image of the homomorphism we consider is the whole $S O(N), G_{2}$ or $\operatorname{Spin}(7)$.

The groups enumerated in Theorem 6.2 appear in the context of entanglement in the paper of Klyachko [20]. This is not entirely accidental. 'Systems in which all unstable states are coherent' considered by Klyachko in his paper can be, in fact, equivalently characterized by our Theorem 6.1. 


\section{Acknowledgments}

We gratefully acknowledge supports from the Polish Ministry of Science and Higher Education through the project no. N N202 090239 and the Deutsche Forschungsgemeischaft through the grant SFB-TR12. We thank Jan Gutt, Mikołaj Rotkiewicz, and Adam Sawicki for very fruitful hints and comments.

\section{References}

[1] Perelomov A 1986 Generalized Coherent States and Their Applications (New York: Springer)

[2] Kuś M and Bengtsson I 2009 "Classical" quantum states Phys. Rev. A 80022319

[3] Klauder J R and Skagerstam B-S (editors) 1985 Coherent States: Applications in Physics and Mathematical Physics (Singapore: World Scientific)

[4] Delbourgo R and Fox J R 1977 Maximum weight vectors possess minimal uncertainty J. Phys. A 10 L233-5

[5] Kotowski, M Kotowski M and Kuś M 2010 Universal nonlinear entanglement witnesses Phys. Rev. A $\mathbf{8 1} 062318$

[6] Lichtenstein W 1982 A System of Quadrics Describing the Orbit of the Highest Weight Vector Proc. Amer. Math. Soc. 84 605-8

[7] Giraud O, Braun P and Braun D 2008 Classicality of spin states Phys. Rev. A 8042112

[8] Wooters W K 1988 Entanglement of Formation of an Arbitrary State of Two Qubits Phys. Rev. Lett $802245-48$

[9] Eckert K, Schliemann J, Bruss D and Lewenstein M 2002 Quantum correlations in systems of indistinguishable particles Ann. Phys. 299 88-127

[10] Hall B 2003 Lie groups, Lie algebras, and representations: an elementary introduction (New York: Splinger)

[11] Barut A and Raczka R 1980 Theory of group representations and applications (Warszawa: PWN)

[12] Bengtsson I and Życzkowski K 2006 Geometry of Quantum States (Cambridge: Cambridge Univ. Press)

[13] Uhlman A 2010 Roofs and Convexity Entropy 12 1799-1832

[14] Grabowski J, Kuś M and Marmo G 2005 Geometry of quantum systems: density states and entanglement J. Phys. A: Math. Gen. 3810217

[15] Mintert F, Carvalho A R R, Kuś M and Buchleitner A 2005 Measures and dynamics of entangled states Phys. Rep. 415 207-59

[16] Alex A, Kalus M, Huckleberry A and von Delft J 2011 A numerical algorithm for the explicit calculation of $S U(N)$ and $S L(N, \mathbb{C})$ Clebsch-Gordan coefficients J. Math. Phys. 52023507

[17] Adams J F 1996 Lectures on Exceptional Lie Groups (The University of Chicago Press)

[18] Montgomery D and Samelson H 1943 Transformation Groups of Spheres Ann. Math. 44 454-70

[19] Olmos C 2005 A geometric proof of the Berger Holonomy Theorem, Ann. Math. 161 579-88

[20] Klyachko A 2007 Dynamic symmetry approach to entanglement (Proceedings of the NATO Advanced Study Institute on Physics and Theoretical Computer Science, IOS Press, Amsterdam), arXiv 0802.4008 